\title{
La configuración habitacional dispersa en tierras secas no irrigadas como estrategia frente a la desertificación. El caso de Lagunas del Rosario, Mendoza, Argentina
}

\author{
Scattered Housing Configuration in non Irrigated Dry \\ Lands as a Strategy to Combat Desertification. \\ The Case of Lagunas del Rosario, Mendoza, Argentina
}

Matías José Esteves ${ }^{\mathrm{I}}$

\section{Resumen}

El noreste de la provincia de Mendoza, en Argentina, se emplaza en un ecosistema de tierras secas con fuertes procesos de desertificación, con acotada disponibilidad de bienes naturales y presencia de asentamientos humanos dispersos. Este trabajo busca comprender la configuración dispersa de estos asentamientos en relación con las características ecológicas del territorio, las actividades productivas que en éste se realizan y el papel del Estado. El objetivo radica en analizar este tipo de asentamiento para comprender la importancia de su localización y su posible vinculación con la sustentabilidad. La metodología utilizada fue la observación directa y la realización de entrevistas semiestructuradas a la población. Los resultados muestran que el patrón de asentamiento disperso responde a las características ecológicas y sociales del territorio, que actualmente representa una estrategia para hacer frente a los procesos de desertificación en el desarrollo de las actividades productivas. Las conclusiones señalan la relevancia de considerar factores sociales, económicos y ecológicos de forma articulada en el abordaje de estos asentamientos y la importancia de la interacción entre el Estado y la población local para alcanzar la sustentabilidad y hacer frente a la desertificación.

Palabras clave: asentamientos humanos dispersos; tierras secas no irrigadas; sustentabilidad; desertificación

\footnotetext{
I Doctorado en Arquitectura y Urbanismo por la Universidad Nacional de San Juan, Argentina. Becario postdoctoral del Instituto de Ciencias Humanas, Sociales y Ambientales (Incinusa) del Consejo Nacional de Investigaciones Científicas y Técnicas (ConiceT), Argentina. Líneas de interés: transformaciones territoriales en zonas rurales, paisaje cultural y arquitectura vernácula. Correo electrónico: mesteves@mendoza-conicet.gob.ar
} 


\section{Abstract}

The northeast of the province of Mendoza (Argentina) is located in the drylands ecosystem, characterized by severe desertification, limited availability of natural resources and scattered human settlements. This article seeks to understand the scattered configuration of the settlements in relation to the ecological characteristics of the territory, the productive activities undertaken and the role of the state. The aim is to analyze this type of settlement to understand the importance of its current location and its link with sustainability. The methodology used was direct observation and semi-structured interviews with the local population together with bibliographic information. The results show that the scattered settlement pattern reflects the ecological and social characteristics of the territory and currently constitutes a strategy for dealing with desertification in the undertaking of productive activities. The conclusions highlight the importance of considering social, economic and ecological factors in an articulated manner in the approach to these settlements and the importance of the interaction between the state and the local population for achieving local sustainability and addressing desertification.

Keywords: scattered human settlements; not irrigated dry lands; sustainability; desertification

\section{Introducción}

La provincia de Mendoza se emplaza dentro del ecosistema de tierras secas, en la diagonal árida sudamericana y presenta clima árido y precipitaciones que no superan los $240 \mathrm{~mm}$ anuales. Dentro del territorio provincial se encuentran dos zonas diferenciadas: por un lado, las tierras secas irrigadas - oasis—, es decir los territorios con mayor acceso y disponibilidad de agua superficial y por ende al riego con posibilidad de cultivo, donde se asienta el $95 \%$ de la población. Estos territorios representan el $3 \%$ de la superficie provincial. Por otro lado, se encuentran las tierras secas no irrigadas que conforman el $97 \%$ de la superficie de la provincia (Gudiño, 2017) y se las define como "espacios conformados por una población dispersa organizada principalmente bajo unidades familiares de autosubsistencia llamados puestos. La principal actividad económica de sus pobladores está constituida por la cría extensiva de ganado caprino y bovino" (MIOPyV, 2014: 42). Las tierras secas no irrigadas se caracterizan por la falta de agua superficial, signadas por procesos de pobreza, pérdida de identidad cultural, afectada ambientalmente por el avance de la desertificación y por aportes estatales discontinuos y desarticulados (Torres et al., 2005; MIOPyV, 2014). Ciertamente, mientras el oasis se asoció al progreso y al uso intensivo y apropiación del agua su- 
perficial, las tierras secas no irrigadas fueron consideradas como antítesis del oasis, rotuladas de "desierto" y asociadas con la desertificación.

Se comprende como la desertificación a "la degradación de las tierras de zonas áridas, semiáridas y subhúmedas secas resultante de diversos factores, tales como las variaciones climáticas y las actividades humanas" (ONU, 1994: 4). Es un proceso mediante el cual las tierras afectadas pierden su capacidad productiva. Entre los factores antrópicos que inciden en la desertificación destacan el sobrepastoreo, el régimen de tenencia de la tierra y la tala indiscriminada, a lo que se suman las variaciones climáticas con sequías recurrentes y prolongadas (Morales, 2005). Una de las causas directas de la desertificación es el manejo inapropiado de la dotación de recursos de un territorio (Granados-Sánchez et al., 2013). Entre las causas indirectas figuran las deficiencias en la distribución del bienestar en las zonas secas, así como las fallas de los mercados, las instituciones y las políticas públicas (Alfaro, 2005).

Se encuentran diversos antecedentes a nivel mundial que abordan la desertificación en tierras secas y su amplia problemática conexa. En estos trabajos se analizan las principales causas que inciden en este fenómeno, tanto climáticas como antrópicas y su combinación (Ejarque, 2009; FAO/LADA, 2011; Abraham et al., 2013; Maestre Gil, 2011). En Latinoamérica se encuentra una vasta producción en el tema, con la generación de indicadores para su medición y control (Correa Moreno, 2015; Troyo Diéguez et al., 2014) y la evaluación de casos comparados que consideran la situación de los países de la región con características físicas y problemáticas similares (Morales, 2012; Abraham et al., 2013; Oliveira et al., 2014). Otros textos se enfocan en la evaluación de la interacción entre la desertificación y la sociedad en un territorio determinado y recuperan las perspectivas de la población local para la puesta en marcha de acciones más concretas (Osorio y Oyarce, 2010; Garay Romero y Cabrero Diéguez, 2013; Erazo y Garay Flühmann, 2011).

Específicamente en Mendoza y al interior de las tierras secas no irrigadas del noreste de la provincia, se encuentran investigaciones donde se evalúa y analiza la desertificación desde una mirada cuantitativa mediante el empleo de indicadores físico-biológicos y socioeconómicos (Rubio, 2015; Abraham y Salomón, 2006). También resaltan aportes desde disciplinas específicas que estudian el manejo de los recursos forestales (Cony, 1995) y la relación entre la carga animal y la cobertura vegetal con la desertificación y las acciones de sobrepastoreo (Guevara et al., 1995). Otros textos se valen de una mirada interdisciplinar con vistas a explicar tanto la pluriactividad en el sector como estrategia para asegurar la reproducción social de los pobladores (Torres, 2008; D’Amico et al., 2013), como algunas prácticas, saberes y estrategias que despliega la población local para el desarrollo de la vida diaria en condiciones de restricción hídrica (Pietragalla y Corso, 1994; Katzer et al., 2017). 
No obstante, la vasta documentación sobre la desertificación, se encuentra en menor medida el análisis de las formas de configuración de los asentamientos humanos en territorios de tierras secas no irrigadas del noreste de Mendoza que son afectados por la desertificación. Si bien históricamente se registra un patrón de ocupación disperso del territorio con vistas al mejor aprovechamiento del medio (Prieto, 1998), actualmente con los procesos de desertificación que se han venido incrementando desde mediados del siglo XX, se ha reducido considerablemente la disponibilidad de bienes naturales. A su vez, desde esa época se registra el desarrollo de "poblados" formados en sectores determinados del territorio en función del lugar donde se canalizaban las acciones estatales, lo que ha provocado que actualmente se encuentren poblados de no más de 40 unidades habitacionales donde se concentran servicios e infraestructura básica. ${ }^{1}$ No obstante el desarrollo de los poblados, se continúan registrando asentamientos humanos localizados de forma dispersa.

Bajo este marco surge la pregunta: ¿por qué los pobladores mantienen el patrón de asentamiento disperso en un territorio con acotada disponibilidad de bienes naturales? Además, en diversos textos a los mismos pobladores se les considera causantes de la desertificación o bien son acusados de provocar su intensificación (Abraham y Salomón, 2014). Con base en lo anterior nos preguntamos ¿cómo interpretan los pobladores los cambios en el territorio en relación con los procesos de desertificación y qué estrategias —en relación con la configuración de los asentamientos humanos- despliegan para adaptarse a esos cambios?

Se sostiene que el patrón de asentamiento humano disperso representa una estrategia para hacer frente a la desertificación, ya que los pobladores encuentran en esta forma de configuración de los asentamientos disponibilidad de forrajes y agua para el desarrollo de sus actividades productivas y su vida diaria, evitando la competencia con sus vecinos por los bienes naturales disponibles. Además, se sostiene que esta forma de asentamiento se vincula con la sustentabilidad, ya que la configuración habitacional dispersa implica el conocimiento del estado del ecosistema y resulta en una mejor forma de relacionarse con el medio natural y sus características. El objetivo este trabajo es analizar el patrón de asentamiento disperso con vistas a comprender la importancia de su localización e indagar respecto a su sustentabilidad en un territorio afectado por la desertificación. Mediante este trabajo se busca entender y enriquecer las actuales formas de configuración de los asentamientos humanos que se encuentran en tierras secas no irrigadas, además de rescatar las perspectivas de la población local.

\footnotetext{
${ }^{1}$ En los poblados se dispone de red eléctrica monofilar inaugurada en 1999 y de una red de agua denominada "acueducto del desierto", que si bien no presenta caudal continuo, facilita el acceso de la población al agua. También se encuentran escuela, centro de salud y registro civil.
} 


\section{Marco conceptual}

Se entiende el concepto de territorio como el binomio naturaleza-cultura y como una construcción social (Benedetti, 2011). Al respecto, Bifani (1999: 31) expresa que "la naturaleza sufre siempre la acción transformadora del hombre y a su vez lo afecta y determina en un proceso dialéctico de acciones e interacciones". En el mismo sentido, Sánchez (1991) aboga por la naturaleza como variable a analizar frente a los estudios sociales y no sólo considerarla como soporte físico inerte, dado que los actores interactúan con ésta de forma permanente. Una de las características distintivas del territorio es su dinamismo, es decir que no se comprende como una realidad acabada sino que se encuentra en constante construcción (Raffestin, 2011), lo que implica considerar la interacción entre tiempo y espacio en su análisis (Haesbaert, 2006).

Esta conceptualización del territorio enriquece el abordaje de la sustentabilidad, ya que implica la consideración de factores sociales y económicos en relación directa con los aspectos ecológicos. Justamente, las problemáticas ecológicas se manifiestan en las formas en que la sociedad o un grupo social se relaciona con la naturaleza para construir su hábitat y generar procesos productivos (Galafassi, 2004). De acuerdo con diversos autores, la sustentabilidad consiste en la adaptación de la sociedad a un factor restrictivo: la capacidad del ecosistema para asumir la presión humana sin que se degrade de forma irreversible (Naredo, 2001; Dimuro Peter, 2009), prestando especial atención a los límites y potencialidades de la naturaleza y a la complejidad de la dialéctica naturaleza-cultura (Bifani, 1999; Leff, 2007).

A su vez, la sustentabilidad también implica considerar el dinamismo del territorio. Es decir que no se considera a la sustentabilidad como un estado de equilibrio a alcanzar y mantener en el tiempo de forma invariable (Jiménez Herrero, 2002) sino que por el contrario, desde el dinamismo del territorio, el desafío se plantea en las adaptaciones a las sucesivas transformaciones. Bifani (1999) sostiene que las transformaciones del territorio pueden originarse por causas internas o externas a un determinado territorio y por ello aboga por el análisis de la sustentabilidad desde diversas escalas temporales y espaciales para evitar sesgar una parte de la realidad. Se coincide con Galván-Miyoshi, Masera y López-Ridaura (2008: 17) cuando sostienen que "los sistemas sustentables son aquellos que "permanecen cambiando", ya que expresan la capacidad de ser productivos, de autorregularse y de transformarse, sin perder su funcionalidad". En esta dinámica se hace referencia también a los conceptos de resiliencia y adaptabilidad. La resiliencia en el marco de la interacción naturaleza-cultura o, como denominan diversos autores, en "sistemas socioecológicos" (Salas Zapata et al., 2012; Balvanera et al., 2017) se refiere a "la capacidad del sistema para absorber perturbaciones y mantener sus funciones" (Balvanera et al., 2017: 142) o mantener el potencial 
productivo sin degradarse de forma irreversible y por lo tanto, alcanzando un estado de equilibrio (Acevedo-Osorio et al., 2017).

Pero este "estado de equilibrio" no hace referencia a un único estado a alcanzar sino que, por el contrario, existen diversos grados de equilibrio y depende principalmente de cómo actúa la población. En este sentido, la adaptabilidad hace referencia a "la capacidad que poseen los afectados para tomar las decisiones que llevan a procesos de gobernanza y autogestión (...) se trata de promover estrategias que contribuyan a generar adaptaciones sustentables, realistas en el contexto de las restricciones propias del sistema" (Balvanera et al., 2017: 143). Por ello, destacan diferentes estrategias para sobreponerse a las perturbaciones y donde pueden figurar diversos grados o estados de equilibrio según las acciones adaptativas que despliegue la población (Acevedo-Osorio et al., 2017). Se trata de promover estrategias acordes al contexto local y por ello diversos autores sostienen que es imprescindible construir marcos de análisis específicos y adecuados para cada contexto socioeconómico y según las características del ecosistema natural.

Interesa hacer referencia a dos versiones del concepto de sustentabilidad que diversos autores consideran que se desprenden de la economía, aunque se remarca que se articulan indefectiblemente con aspectos sociales y ecológicos. Una de estas versiones es la sustentabilidad débil, que se ubica dentro del paradigma de la economía estándar y la confianza en la tecnología. Desde otro ángulo, se encuentra la sustentabilidad fuerte que se vincula con la termodinámica y la ecología. La sustentabilidad débil se apoya en la combinación del capital natural y el capital de formación humana. El capital natural constituye la disponibilidad y flujos de bienes naturales que ingresan a una determinada sociedad, mientras que el capital de formación humana implica la disponibilidad de capital monetario, de tecnología y/o personal capacitado. Esta noción se asienta en el principio de sustitución que implica que los recursos que se agotan pueden ser sustituidos ilimitadamente por el capital de formación humana (Bifani, 1999). Es decir, sustituir un recurso por otro que realice la misma función o reemplazar el trabajo por capital.

Daly (2007) señala que el principio de sustitución resulta destructivo e insuficiente frente a problemas como la contaminación o el calentamiento global, donde la tecnología o inversión monetaria no podrían sustituir la capacidad depuradora y recicladora de la naturaleza. La sustentabilidad fuerte, por el contrario, se basa en que el sistema socioeconómico sea dependiente del ecosistema natural en una articulación permanente, considerando ambos como sistemas dinámicos (Bifani, 1999; Martínez Alier, 2006). Esta noción reconoce que los sistemas naturales son diferentes, con distinta capacidad de carga y disponibilidad de bienes naturales y por esta razón se aboga por la regionalización de la economía para alcanzar la sustentabilidad. Esta corriente se 
propone revalorar los aspectos locales, contraponiéndose a los planteos globalizadores y al crecimiento económico incontrolado.

En las tierras secas no irrigadas del noreste de Mendoza, los asentamientos humanos se definen por ser a la vez unidad de habitación y de producción, en donde se vincula la vida familiar con el trabajo. No se hace referencia a una construcción única, sino a una conjunción de elementos que combinan diversas funciones y actividades (Pastor, 2005). Los elementos irreductibles que se encuentran en los asentamientos humanos son: la unidad de habitación, los corrales (elementos de la actividad productiva principal centrada en la cría de ganado caprino) y los dispositivos de acceso al agua subterránea centrados en los pozos jagüel o aguada y el pozo balde. Estos asentamientos son la unidad económica-productiva más típica de la zona de estudio (Torres et al., 2005).

\section{Metodología}

Se utilizaron métodos cualitativos: la observación directa y entrevistas semiestructuradas para la construcción de datos a partir de un caso de estudio. De acuerdo con el problema de investigación se seleccionó como caso el distrito de Lagunas del Rosario, emplazado en el departamento de Lavalle, en el noreste provincial. Este territorio se encuentra habitado históricamente por una comunidad indígena Huarpe. A su vez, el sitio presenta grandes transformaciones territoriales acontecidas, en gran parte, por su localización periférica respecto al oasis norte — principal zona irrigada en la provincia de Mendoza - y desde la relación histórica entre zonas no irrigadas y oasis, lo que implicó también un abordaje multiescala. En cuanto al ecosistema natural, el sitio se emplaza en el árido mendocino sometido a fuertes procesos de desertificación que inciden en las actividades productivas de subsistencia que despliegan los pobladores.

La observación directa se utilizó con la finalidad de examinar las formas en que se configuran los asentamientos humanos dispersos (materiales empleados, cantidad de unidades de habitación, dispositivos de acceso al agua) y las actividades productivas que se realizan (tipos de actividades y elementos o construcciones que se utilizan para su desarrollo). Mediante las entrevistas semiestructuradas de carácter flexible, dinámico y abierto (Taylor y Bogdan, 2008) realizadas a la población local se buscó comprender aspectos que no se pueden observar de forma directa vinculados con la localización dispersa de los asentamientos, datos sobre su cotidianeidad, sus percepciones respecto a las transformaciones del territorio y la mano de obra utilizada en el desarrollo de sus actividades productivas. La selección de los entrevistados se realizó por el método de bola de nieve. Aunque se visitaron todos los asentamientos humanos dispersos en Lagunas del Rosario, se trabajó con aquellos en donde fue posible localizar a los entrevistados (ya que muchos migran por 
trabajos temporales). También se realizaron entrevistas semiestructuradas a empleados públicos de diversas instituciones gubernamentales que trabajan en el sector de estudio, a fin de comprender cómo el Estado encara las políticas públicas en el sector.

\section{Naturaleza y cultura en Lagunas del Rosario}

Lagunas del Rosario se localiza en el noreste de la provincia de Mendoza, a $110 \mathrm{~km}$ de la capital provincial, en el departamento de Lavalle (Figura 1). La zona de estudio presenta clima de tipo desértico-semidesértico y en este sitio se ubican algunos de los lugares más áridos de la Argentina (Abraham y Prieto, 1999). El régimen de precipitaciones se caracteriza por la presencia de tormentas convectivas de verano, aunque anualmente se registran en el área entre $80 \mathrm{~mm}$ a $150 \mathrm{~mm}$ anuales de lluvia.

\section{Figura 1. Mapa del departamento de Lavalle con asentamientos dispersos y el sector de estudio}

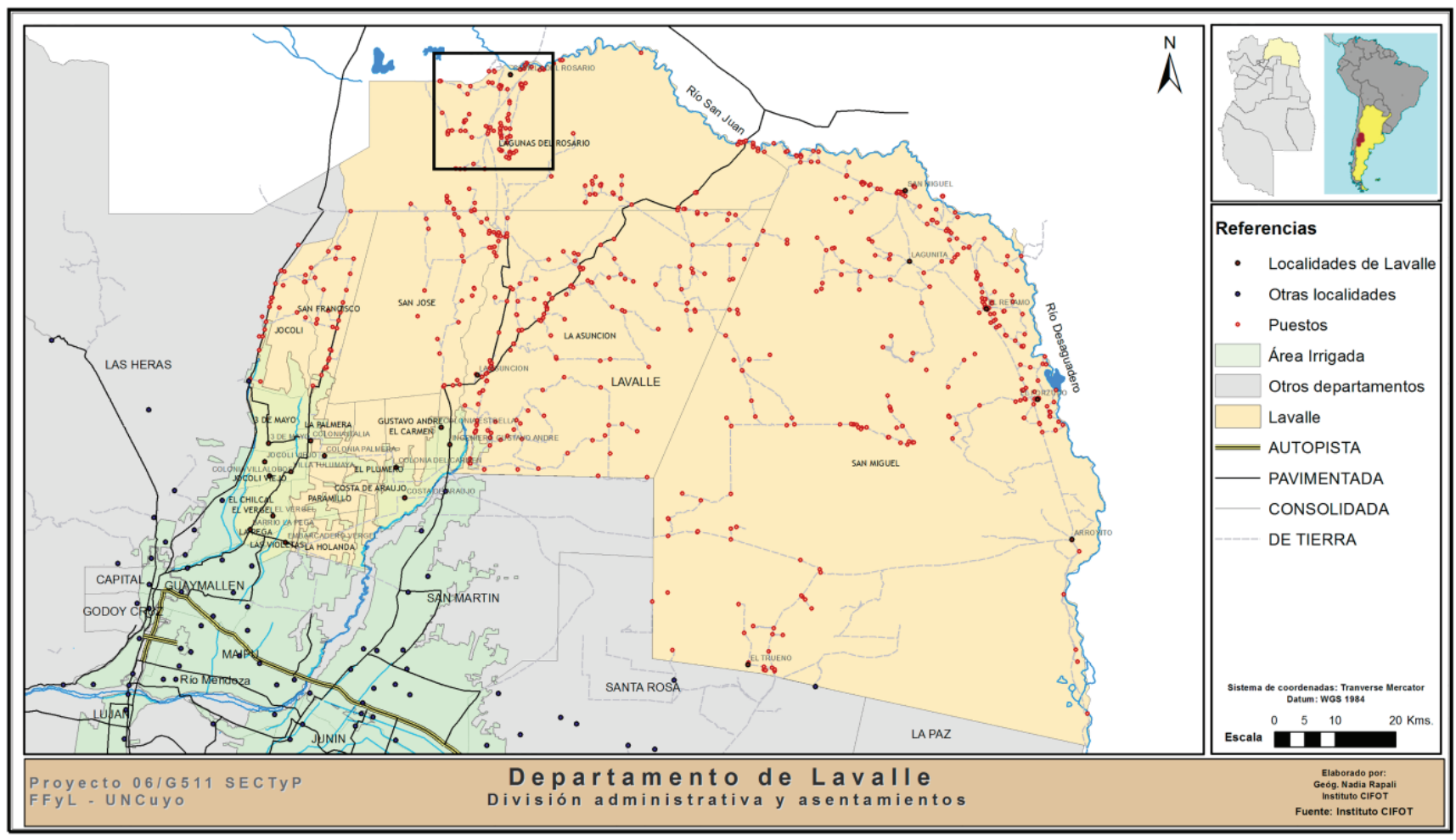

Fuente: Miranda Gassull y Gómez, 2016.

Nota: Obsérvese su ubicación periférica respecto al área irrigada (oasis). 
En relación con la vegetación, el sector de estudio se localiza dentro de la Provincia Fitogeográfica del Monte, donde predominan diversas comunidades arbustivas xerófilas de hoja perenne, encontrándose bosques aislados de algarrobo (Prosopis Flexuosa) acompañado por otros árboles como el chañar (Geoffroea decorticans) y el retamo (Bulnesia retama). Entre los pastos sobresalen Junquillo (Sporobolus rigens) y Coiron (Stipa sp.) (Chiavazza, 2008). Respecto a la disponibilidad de agua, el noreste provincial actualmente carece de escorrentía superficial. Sin embargo, se encuentra en el subsuelo un considerable reservorio de agua a escasa profundidad —entre los seis y diez metros- y aunque presenta mala calidad ${ }^{2}$ facilita el mantenimiento de la vida en la zona.

En Lagunas del Rosario se encuentran dos patrones de asentamientos humanos: por un lado, asentamientos conformados por la presencia de hasta 40 unidades habitacionales agrupadas que conforman pequeñas tramas denominadas "poblados", ${ }^{3}$ con presencia básica de servicios e infraestructura del Estado. Por otro lado, se encuentran asentamientos humanos dispersos por el territorio. Estos últimos, a su vez, se los ha clasificado en dos grupos: el primero involucra a aquellos asentamientos humanos que destacan por la presencia de dos a cinco puestos en el mismo predio, donde los usuarios son familiares directos entre sí y por eso se los ha denominado "asentamientos familiares". El segundo grupo lo integran asentamientos conformados por un único puesto, denominados "asentamientos solitarios" (Esteves, 2016). La diferencia entre ambos grupos radica en que los asentamientos familiares son los de mayor trayectoria temporal y disponen de mano de obra permanente para el desarrollo de las actividades productivas.

Estos asentamientos familiares se encuentran alejados de sus vecinos a una distancia que oscila entre los $2.5 \mathrm{~km}$ a $7 \mathrm{~km}$ y por lo tanto, la disponibilidad de mano de obra al interior del predio es clave para el desarrollo de las actividades productivas y la vida diaria, resaltando la estrategia de agrupamiento familiar. En contraposición, los asentamientos solitarios presentan mano de obra limitada a esa única familia aunque la proximidad a los vecinos (entre $0.7 \mathrm{~km}$ a $4 \mathrm{~km}$ de distancia) propicia relaciones de reciprocidad en diversas situaciones (Figura 2). En ambos casos, se emplazan sobre antiguos cauces de agua o en los costados de los ríos secos, ya que se trata de ubicaciones estratégicas para aprovechar la esporádica presencia de agua de lluvia o bien acceder al agua subterránea a menor profundidad.

\footnotetext{
${ }^{2}$ El agua posee altos niveles de salinidad en combinación con la presencia natural de arsénico (HACRE) (Pastor, 2005). Esta situación afecta la vida de los pobladores y la de sus animales. Algunos pobladores caminan hasta puestos vecinos para conseguir agua de mejor calidad (Grosso Cepparo, 2013). Para enfrentar esta situación, el municipio reparte agua potable a través de camiones cisternas que los pobladores almacenan en tanques de 1100 litros de PVC dispuestos para tal fin (el gobierno repartió un tanque por unidad habitacional). No obstante, en las entrevistas los pobladores comentaron que la repartición de agua en los asentamientos humanos dispersos se realiza cada mes y medio o dos meses.

${ }^{3}$ En Lagunas del Rosario se encuentran dos poblados. El principal se organiza en torno a la Capilla del Rosario, edificio histórico, que data de 1609, que identifica a la zona y a la comunidad Huarpe de Lagunas del Rosario. Este poblado no supera las 35 unidades de habitación. A $4 \mathrm{~km}$ de la Capilla se encuentra otro poblado conformado por 12 unidades de habitación localizadas en torno a la "pista del avión sanitario"; infraestructura que en un momento histórico sirvió para la provisión de elementos, atención médica y registro civil.
} 


\section{Socjedad Ambiente}

$\mathrm{El}$ acceso a todos los asentamientos humanos dispersos se realiza a través de huellas sobre el suelo arenoso - la mayoría en mal estado- que se desprenden del camino principal de ripio que atraviesa el sector (Figura 2). A estas huellas no se les da mantenimiento, lo que dificulta el acceso a la mayoría de los asentamientos humanos dispersos. El camino de ripio que atraviesa el sector es mantenido por el municipio, aunque en diversos tramos presenta malas condiciones para la circulación. Este camino culmina en el poblado de Lagunas del Rosario y por ello el mantenimiento es mínimo ya que sólo se utiliza para circular desde y hacia este sector.

\section{Figura 2. Imagen aérea del sector de estudio con distintos patrones de asentamiento e infraestructura}

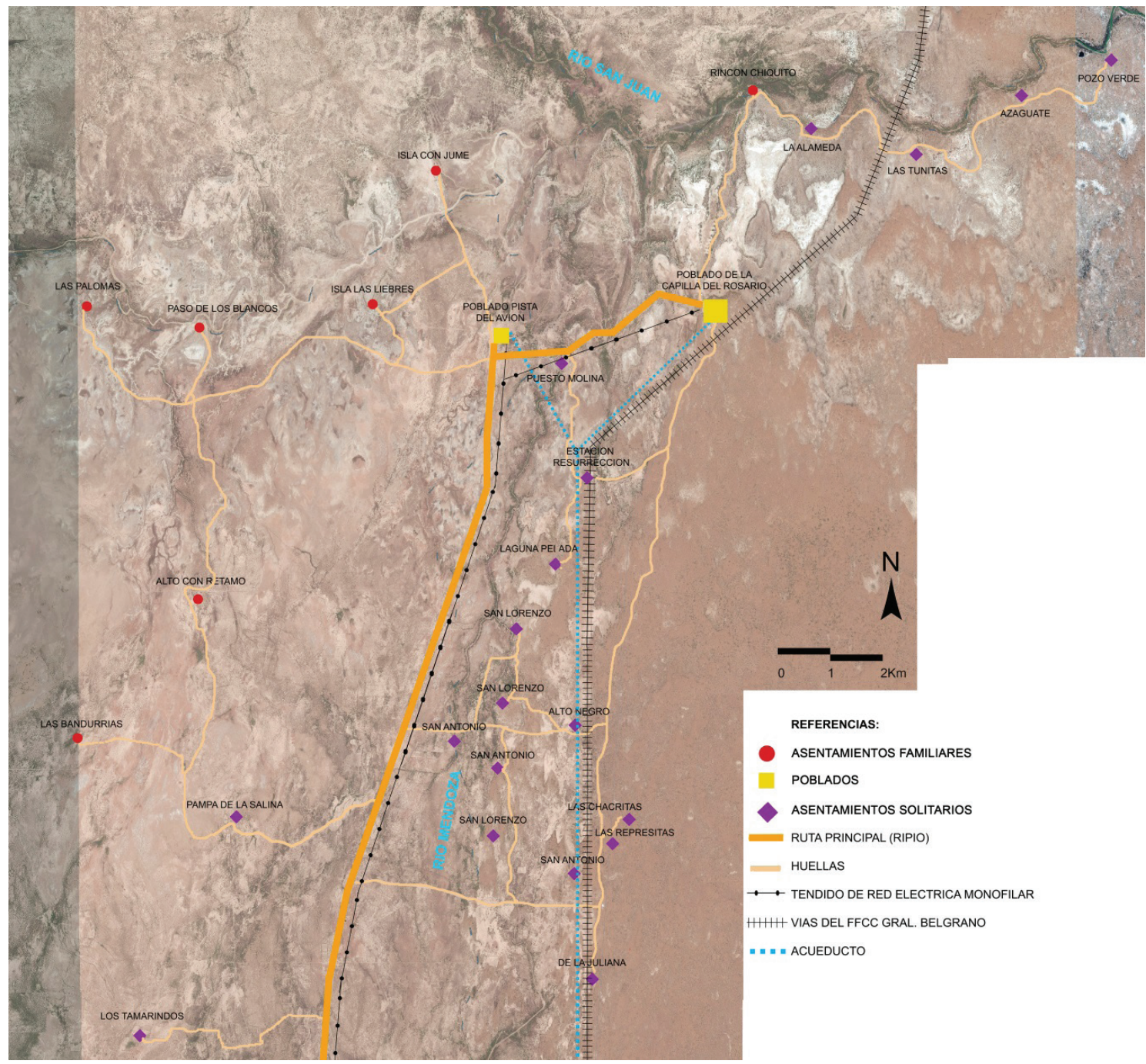

Fuente: Esteves, 2016

Nota: Obsérvese la distancia entre asentamientos familiares y solitarios. 
Respecto a las características socioeconómicas, en las tierras secas no irrigadas del noreste de Mendoza se desarrollan economías de subsistencia, donde la actividad productiva principal es la cría de ganado caprino a escala familiar. También se registran en menor medida otras actividades como la venta de guano, la recolección de junquillo y algarrobo ${ }^{4}$ y la realización de artesanías. Los productos se destinan principalmente al autoconsumo y en menor medida a la venta. También se registran actividades temporales que implican migraciones hacia fincas cercanas ubicadas dentro de las zonas irrigadas.

Interesa remarcar que el noreste de Mendoza se encuentra habitado históricamente por comunidades indígenas Huarpes. Recién en el año 2010 y por medio de la ley núm. 6920 el gobierno de Mendoza procedió al reconocimiento de la preexistencia étnica cultural del pueblo Huarpe Milcallac organizado en 11 grupos de descendientes, garantizando el respeto a su identidad cultural. En el mismo año, la comunidad Huarpe de Lagunas del Rosario recibió la titularidad de 72 mil hectáreas como bien comunitario, con lo cual se reconoció su forma de acceso comunitario a los bienes naturales. Es decir, que si bien históricamente se ha ocupado el territorio de forma comunitaria, hasta hace poco tiempo no era reconocido como tal.

$\mathrm{Al}$ respecto y en comparación con el modelo implantado en el oasis, Katzer (2010: 10) comenta que "la elaboración histórica del modo de tenencia de la tierra indígena, fundada en los derechos de sus antepasados, con una concepción de posesión compartida de la tierra y sostenida tradicionalmente por una estructura de parentesco y de relaciones de reciprocidad, en la cual el grupo y el medio se asumen como un todo, se vio interrumpida por un modelo de dominio definido por el parcelamiento del territorio y la utilización del medio de manera privada". Por ello, el reconocimiento de la tenencia comunitaria resultó en una acción significativa para la comunidad local, que marca un hito en el reconocimiento no sólo de otras formas de tenencia de la tierra sino también de las estrategias de los pobladores para acceder a la naturaleza.

\section{Resultados y discusión}

A continuación se expone un recorrido histórico del sitio en cuanto a la disponibilidad de agua superficial y a las actividades productivas que permitían la presencia de agua, para luego contextualizar los cambios en el territorio, las estrategias que despliegan los pobladores para adaptarse a esos cambios e indagar respecto a su localización dispersa.

\footnotetext{
${ }^{4}$ El fruto del algarrobo se utiliza para autoconsumo como alimento y en la elaboración de harina (Roig et al., 1999). Además los pobladores la recolectan con la finalidad de alimentar también al ganado, principalmente en época invernal donde la disponibilidad y calidad del forraje natural disminuye.
} 
Históricamente los cauces de agua superficial generaron en la zona un extenso sistema hidrográfico, denominado Lagunas de Guanacache, ${ }^{5}$ que facilitó la ocupación humana a pesar de las condiciones de aridez. En torno a las lagunas giraba la vida productiva y social de la población local, donde la presencia de masas de agua favoreció la pesca y permitió el desarrollo de la agricultura como actividades productivas principales de la zona (Roig et al., 1999), y donde los productos obtenidos eran comercializados en la ciudad de Mendoza (Masa, 1990). De acuerdo con Prieto (1998) la localización de los asentamientos en el sector de estudio a finales del siglo XVII respondía a la cercanía a cursos de agua superficial y la posibilidad de contar con tierras cultivables. En este sentido y de acuerdo con las condiciones de aridez del territorio, hacia fines del siglo xIX se registra un asentamiento ribereño o costero y disperso (Abraham y Prieto, 1981). Efectivamente, la forma de organización dispersa respondía a las características ecológicas, donde "el modelo seleccionado para la mejor utilización de los recursos requería la dispersión, la agricultura no era posible en gran escala por la naturaleza del terreno" (Prieto 1998: 61).

Las principales transformaciones del territorio se producen desde finales del siglo XIX, donde adquiere gran protagonismo en la zona del oasis el modelo productivo centrado en el cultivo intensivo de la vid, el cual se consolidó como el principal motor del desarrollo en toda la región (Richard Jorba, 2000). Este modelo implicó mayor apropiación y uso de agua ${ }^{6}$ en la zona de los oasis, lo que significó menor caudal de agua hacia las lagunas, las cuales fueron reduciendo considerablemente su tamaño. Esta situación, sumada a un periodo de sequía que afectó a la región de 1901 a 1915 (Abraham y Prieto, 1999), provocó la desecación de las lagunas en la década de 1940 (Roig et al., 1999), aunque recibían aportes de agua de forma ocasional en épocas estivales a causa del deshielo (Abraham y Prieto, 1999). La década de 1980 es la fecha en que señalan los pobladores en las entrevistas que dejó de llegar agua de forma definitiva.

El crecimiento y progreso económico del oasis se complementó con la extracción de vegetación de las zonas no irrigadas. Se aprovechó la madera del bosque nativo para satisfacer la demanda de leña de la población en aumento en el oasis, el uso y construcción de las vías del ferrocarril y el armado de postes para la instalación de las nuevas hectáreas cultivadas por la industria vitivinícola (Abraham y Prieto, 1999). El exceso en la apropiación del bosque nativo provocó la pérdida de grandes masas forestales. La falta de agua superficial cada vez más evidente en combinación con la tala indiscriminada propició procesos de desertificación que afectaron al ecosistema y con ello a las actividades productivas que de manera histórica se desarrollaban en la zona —agricultura y

\footnotetext{
${ }^{5}$ La confluencia de los ríos Mendoza, San Juan y Desaguadero constituía el complejo hídrico integrado por diversas lagunas encadenadas que se comportaban como grandes espejos de agua, por ello en varios textos se destaca que el sector cuenta con una gran biodiversidad (Rosales, 1877; Roig et al., 1999); lo cual da cuenta de que la realidad del ecosistema natural era diferente al actual.

${ }^{6}$ La vitivinicultura necesita entre 700 a $800 \mathrm{~mm}$ de agua para su desarrollo. Al ser bajas las precipitaciones a escala provincial, la actividad es altamente dependiente del agua superficial del rio (Torres et al., 2003).
} 
pesca - como economía de subsistencia. La cría de cabra adquirió protagonismo por ser la actividad que mejor se adaptó a las nuevas condiciones ecológicas, con pastos duros y agua subterránea de mala calidad.

A continuación se describen los asentamientos humanos dispersos y los elementos irreductibles que lo componen —unidad de habitación, corrales y dispositivos de acceso al agua subterránea-. Esta descripción resulta importante con el fin de comprender su organización y funcionamiento.

En todos los asentamientos humanos analizados, la unidad de habitación se conforma por una serie de locales contiguos comunicados directamente entre sí (Pastor, 2005) que presentan una galería abierta que se localiza en mayor medida hacia el norte. Para su materialización se utilizan principalmente materiales naturales: barro sin cocer en muros —adobe o quincha- y cubierta de torta de barro. El uso de estos materiales responde a motivos económicos (ya que se encuentran en el medio, además del costo que supone el traslado de materiales desde los poblados urbanos hasta el asentamiento disperso), a motivos sociales (ya que los saberes asociados con estas técnicas de construcción se transmiten entre generaciones), y a motivos funcionales, ya que resultan más adecuados frente al clima. Pero también se observa la incorporación en menor medida de materiales industrializados como son el ladrillo, la chapa acanalada de zinc y láminas de polietileno. La incorporación de nuevos materiales tiene que ver con disminuir las tareas de mantenimiento que implica el uso de materiales naturales y con asociar éstos con mejores posibilidades de "progreso" y estatus social (Esteves, 2014). En los "asentamientos familiares", las unidades de habitación se localizan entre sí a una distancia que oscila entre los 50 y los 200 m (Figura 3).

La principal actividad productiva en todos los asentamientos humanos dispersos es la cría de ganado caprino, y por ello destaca la presencia de varios corrales, que son de forma rectangular y sus laterales se construyen con postes de madera local en combinación con enramadas y chapa acanalada de zinc. En todos los casos analizados, se localizan respecto a la unidad de habitación a una distancia que oscila entre los 50 y 300 m. Cada unidad de habitación posee sus propios corrales (de uno a tres), que muchas veces se construyen de forma contigua para compartir un lateral y disminuir la mano de obra y disponibilidad de materiales (Figura 3). La cantidad y dimensiones de los corrales dependen del tamaño de la majada de la cual dispone cada asentamiento, que oscila entre las 70 y 200 cabezas. El número de cabezas de ganado depende principalmente de la mano de obra disponible al interior de cada asentamiento y la distancia respecto a los vecinos (por la "competencia" por los bienes naturales). Así, los asentamientos familiares presentan mayor cantidad de cabezas, mientras que los asentamientos solitarios presentan una menor cantidad. 
Figura 3: Fotografías aéreas y esquemas de los dos tipos de asentamientos humanos dispersos con elementos que los componen

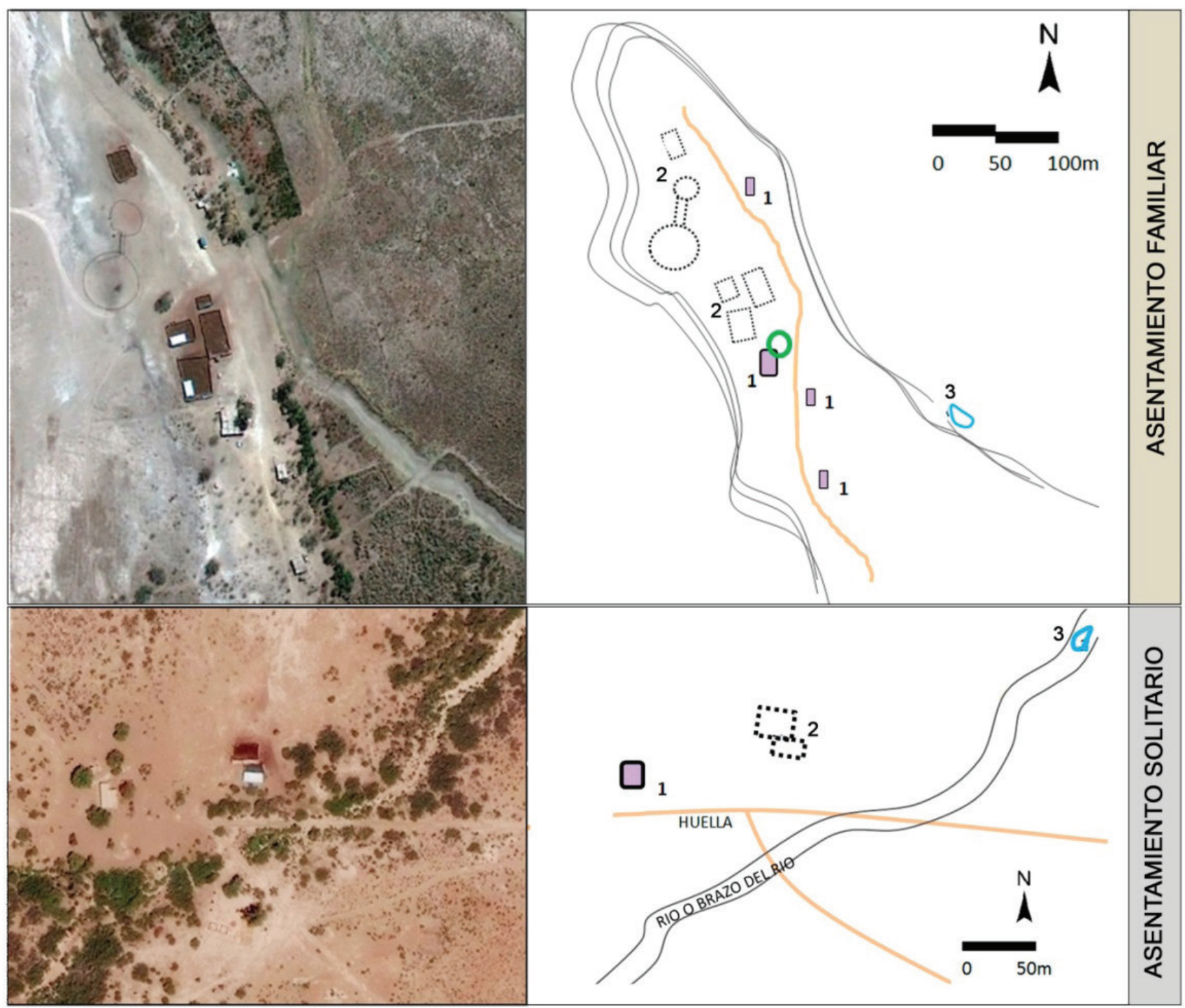

Fuente: elaboración propia en base a Google Earth.

Nota: 1- Unidad de habitación; 2- Corrales; 3- Pozo Jagüel o aguada.

De acuerdo con autores como Rubio (2015), el área de pastoreo alrededor del asentamiento ronda entre los 2 y $4 \mathrm{~km}$ considerando la distancia en que las cabras se desplazan. Además, esta distancia fue constatada en el trabajo de campo desde que fue consultada la población local, quienes la definieron según el recorrido aproximado en el cual se mueven diariamente las cabras y la distancia que transitan los pobladores para la recolección de leña y otros recursos, como la algarro- 
ba. La actividad pecuaria se realiza de forma extensiva, es decir que no existen alambrados que delimiten los campos. Esta forma de pastoreo se vincula con la tenencia comunitaria de la tierra e implica que los animales de cualquier poblador puedan andar por el campo. No obstante esta actividad principal, se reconocen también diversas actividades secundarias, encontrándose la venta de guano para su uso en fincas de las zonas irrigadas, la realización de artesanías, la recolección de junquillo y la realización de trabajos extraprediales en las fincas cercanas ubicadas en las zonas irrigadas. Torres expone claramente la importancia de la pluriactividad en el noreste de Mendoza: "en la medida en que producen diferentes bienes, las unidades domésticas multiplican las oportunidades de venta y, al unísono, reducen el riesgo que significaría dejar al amparo de un único producto y mercado, la reproducción social de la familia” (Torres, 2008: 210).

Entre los dispositivos para el acceso al agua subterránea sobresale el pozo balde que se utiliza para extraer agua para realizar las tareas domésticas y el pozo jagüel o aguada que se emplea para el abrevado del ganado. Interesa focalizarse en este último por su relación con la actividad productiva principal de los asentamientos. El pozo jagüel consiste en una rampa excavada hasta alcanzar la primera napa de agua (entre los 6 y $10 \mathrm{~m}$ de profundidad). En la zona de estudio estos dispositivos se realizan en los fondos de las lagunas, en los brazos secos de los ríos o en zonas deprimidas con la finalidad de encontrar agua a menor profundidad y reducir las tareas de excavación. Estos dispositivos son construidos en diversos sectores en torno al asentamiento con la finalidad de asegurar la presencia de agua para las cabras, lo cual se relaciona con la cría extensiva del ganado, donde la majada de cualquier poblador puede hacer uso de los pozos. Por esta razón se construyen entre las familias y a veces con ayuda de los vecinos cercanos, lo que implica su uso compartido y relaciones de reciprocidad.

De acuerdo con los datos obtenidos en las entrevistas, para la construcción y mantenimiento de la unidad de habitación como de los corrales y las aguadas, la mano de obra es principalmente familiar, con ayuda de parientes cercanos o vecinos. Es decir, que se hace referencia a la autoconstrucción. Lo mismo sucede con las actividades productivas ya que son los mismos pobladores quienes se encargan del cuidado y atención de la majada. La participación activa de toda la familia implica la presencia de saberes tradicionales y el aprendizaje a través de la experiencia por medio del ensayo a "prueba y error". Además, en este proceso de participación activa, los mayores traspasan los conocimientos y aprendizajes a los más jóvenes y de esta manera se asegura una continuación respecto a la mejor forma de vincularse con el medio.

Cuando se indagó a los pobladores respecto a la localización del asentamiento, todos los entrevistados coincidieron en señalar que mediante la configuración dispersa aseguran disponibilidad de forraje para el mantenimiento de la actividad productiva principal; es decir, que la 
dispersión es la nota distintiva para el desarrollo de la cría de cabra. Si bien son conscientes de que se encuentran alejados de los poblados (entre 3 y $15 \mathrm{~km}$ de distancia) donde existen servicios e infraestructura básica que brinda el Estado, resulta más importante para ellos asegurar el éxito en las actividades productivas que les permiten el sustento de la vida diaria. Ciertamente, tanto los pobladores de los asentamientos dispersos como aquellos radicados en el poblado reconocen la situación actual del campo y comentan las dificultades que implica el desarrollo de la actividad pecuaria en torno al poblado.

"Pasa que el campo cerca de la capilla [poblado] no sirve nada. Si no hay agua no está bueno el campo. Aparte que son muchos por aquella zona y el campo es menor para la cantidad de animales que tienen. En cambio acá tienen comida y agua" (Poblador, 56 años. Asentamiento humano disperso. Trabajo de campo, 2015).

"Yo tengo mi casa acá y hago artesanías. Mi hermano que tiene el puesto con los animales vive más alejado. Acá en el poblado no vas a ver cabras. Casi la mayoría que tiene cabras están lejos, en los puestos. Yo acá no las pude mantener y vendí las ultimas que tenía" (Pobladora, 62 años. Poblado. Trabajo de campo, 2014)

Efectivamente, en el poblado se encuentra mayor cantidad de personas viviendo en una misma superficie acotada, ${ }^{7}$ lo que implica mayor presión sobre el suelo y por ende mayor competencia por las pasturas. Por ello, en los poblados no sobresale la cría de cabra como actividad principal, sino que predominan empleos en relación con los servicios del Estado, la elaboración de artesanías y trabajos temporales en fincas cercanas ubicadas en las zonas irrigadas.

En vinculación con lo anterior, se encuentra una discusión respecto al grado de sustentabilidad que alcanzan las prácticas ganaderas extensivas, ya que si bien esta forma de pastoreo implica mínima inversión de capital económico y una alta rentabilidad respecto al capital invertido en el corto y mediano plazo, se pone en duda la sustentabilidad a largo plazo (Espejo, 1996). Varios autores exponen que en gran parte de América Latina predominan las prácticas extensivas y con ello figuran diversos estudios que indagan en la aplicación de mejoras a esta práctica ${ }^{8}$ asociadas a la sustentabilidad (Martínez, 2003). Pero la sustentabilidad a largo plazo en territorios con acotada disponibilidad de bienes naturales sumado a procesos de desertificación implica analizar la

\footnotetext{
${ }^{7}$ No se cuenta con el número exacto de personas que habitan en el poblado de la Capilla del Rosario, ya que los datos estadísticos publicados por Organismos Oficiales abarcan todo el Distrito de Lagunas del Rosario. De acuerdo al relevamiento de campo se han registrado alrededor de 35 viviendas en el sector del poblado en torno a la Capilla, con lo cual se estima que habitan alrededor de 140 personas, considerando cuatro por unidad habitacional, en un radio de $300 \mathrm{~m}$.

${ }^{8}$ Las ventajas de las prácticas extensivas involucran el mantenimiento de las poblaciones en zonas rurales, la revalorización de los territorios, y el reconocimiento de la importancia de los bienes naturales a escala local, entre otros (López, 2002).
}

Sociedad y Ambiente, año 7, núm. 18, noviembre de 2018-febrero de 2019, ISSN: 2007-6576, pp. 71-94 
regulación de los límites máximos de cabezas de ganado que podría alcanzar cada poblador en pos de no rebasar la capacidad de carga del ecosistema de forma irreversible.

Si bien se ha expuesto que la configuración habitacional dispersa resulta clave para la disponibilidad de forraje, esta estrategia no se apoya en acuerdos o normas entre los pobladores que regulen la cantidad de cabezas de ganado a disponer para evitar el agotamiento del campo. Tampoco desde las instituciones del Estado se encuentran acciones concretas respecto a la cría de ganado. ${ }^{9}$ En todas las entrevistas realizadas a los pobladores queda de manifiesto que la limitante respecto a la cantidad de cabezas de ganado que disponen deriva de la combinación del estado del campo y la mano de obra disponible. Pero no se encuentra una "reflexión" respecto a la posibilidad de regular la cantidad de cabezas de ganado con vistas a propiciar o atender la recuperación del campo en beneficio de la comunidad o desde el Estado en pos de afrontar la desertificación y mejorar las prácticas ganaderas.

Las estrategias vinculadas con la práctica extensiva de ganado que despliega la población se vinculan con la ausencia de agua superficial y de tecnología o la falta de capital económico por parte de los pobladores para adquirir la tecnología adecuada para regar los campos y con ello realizar las prácticas intensivas que permitan la rotación del ganado y recuperación del campo. Además, esta práctica se sustenta en los cambios en el territorio, que de acuerdo con todos los entrevistados los principales derivan de la acotada disponibilidad de agua superficial debido a la apropiación y el uso intensivo que se ha realizado y que aún se realiza en el oasis. En sintonía, varios autores locales advierten la problemática de acceso diferencial al agua en el oasis en detrimento de los territorios no irrigados (Grosso Cepparo, 2017)

"El gobierno dice emergencia hídrica ${ }^{10}$ pero debería ser emergencia para todos. Entonces no se deberían plantar más viñedos. Pero acá creo que no estamos en equidad igualitaria para todos, todavía falta" (Poblador, 50 años de edad. Asentamiento humano disperso. Trabajo de campo, 2013).

A continuación se expone la relación entre el Estado y la población local, ya que diversos autores consideran clave esta relación para hacer frente a los procesos de desertificación. En las entrevis-

\footnotetext{
${ }^{9}$ Desde el Estado se ha identificado la baja en la producción caprina debido al estado del campo y por ello se realizaron capacitaciones que pretenden mostrar cómo se puede aprovechar el bosque nativo a la vez que favorecer su recuperación. Esto implicó capacitaciones para el armado de escobas a partir de la recolección del junquillo, elaboración de artesanías en madera aprovechando los sobrantes de la poda y la elaboración de harina a partir de la algarroba. De acuerdo a las entrevistas realizadas a empleados públicos, el problema principal de estas acciones fue su falta de continuidad por razones de presupuesto. Se suma también que no se vinculaban directamente con la actividad productiva principal, que es la más redituable para los pobladores.

${ }^{10}$ La provincia de Mendoza enfrenta el octavo año consecutivo de emergencia hídrica. http://www.prensa.mendoza.gov.ar/irrigacion-pronostico-el-8o-ano-de-emergencia-hidrica-y-por-primera-vez-anuncio-medidas-para-enfrentarlo/
} 
tas realizadas a los pobladores y a algunos empleados públicos en vinculación directa con el sector de estudio queda de manifiesto que la mayoría de los pobladores de Lagunas del Rosario se encuentran predispuestos y participan en los proyectos del Estado, sobre todo en aquellos que tienden a implementar mejoras en el ecosistema natural y repercuten directamente en su calidad de vida y en el desarrollo de las actividades productivas. Asimismo los pobladores exponen su preocupación cuando los veterinarios o el Estado se ausentan, ya que reconocen la importancia de contar con los conocimientos técnicos y con una formación permanente en torno a las actividades productivas que realizan y las formas en que podrían hacer frente a nuevos obstáculos. Por ello indican que el problema principal de las políticas en zonas no irrigadas radica en la falta de constancia en su implementación, así como de seguimiento y consideración hacia los pobladores en tanto actores clave en el desarrollo del territorio.

\begin{abstract}
"Antes venían del PSA [Programa Social Agropecuario] y daban capacitaciones. También venía seguido un veterinario. Después con el problema de límites entre provincias dejaron de venir y ahora hay que llamarlos para que vengan" (Pobladora, 40 años. Asentamiento humano disperso. Trabajo de campo, 2015).

"En la zona de Lagunas los pobladores nos han hecho hasta notas pidiendo que por favor se hagan capacitaciones para el manejo y cuidado de la cabra. Pero esto muchas veces se dificulta porque acá te plantean que es un retroceso volver a hacer algunas capacitaciones porque nos dicen que si ya se hicieron en una época, ¿para que lo van a hacer de vuelta? Para mí es una mirada muy cuadrada de la Institución porque la gente y las necesidades van cambiando. Ahora los hijos se encargan del puesto y la gente quiere volver a hacer las capacitaciones" (Entrevista a empleado público. Trabajo de campo, 2015).
\end{abstract}

En los relatos se observa la dependencia directa del grupo social con el Estado y el papel importante que posee este último en las tierras secas no irrigadas. Si bien los pobladores poseen saberes tradicionales que tienen que ver con la mejor forma de adaptarse al medio a través de la "prueba y error" realizados en la práctica cotidiana, los conocimientos técnicos, tecnología y capacitación que podría aportar el Estado serían la clave para abordar la desertificación en el sector de forma conjunta y alcanzar la sustentabilidad a largo plazo de las actividades productivas. En este sentido, se coincide con Abraham y Salomón (2014: 63) cuando expresan que "los pobladores de tierras secas son su mayor recurso. Ellos conocen sus problemas y sus potencialidades y han desarrollado conocimientos, tecnologías y habilidades para producir en situaciones restrictivas". 


\section{Consideraciones finales}

Si bien la Comunidad Huarpe históricamente se ha vinculado de forma comunitaria evitando el individualismo y la competencia por los bienes naturales, esta característica adquiere mayor relevancia con el desarrollo de los procesos de desertificación. Ciertamente, en la tenencia comunitaria de la tierra se apoyan diversas estrategias, como son el pastoreo extensivo y la localización dispersa de los asentamientos humanos. Estas estrategias se vinculan de forma directa con el estado ecológico del sector de estudio, donde el pastoreo sin alambrados implica que el ganado pueda ir hacia diferentes zonas del territorio y evitar que las cabras pastoreen en la misma superficie. En esta situación se asienta también el acceso común al agua subterránea desde la construcción de pozos jagüel o aguadas donde la majada de cualquier poblador puede hacer uso de estos dispositivos para el abrevado del ganado. El acceso comunitario a los bienes naturales también involucra a la vegetación, ya que los pobladores encuentran en el bosque nativo un recurso importante, no sólo por ser fuente de madera y leña, sino también por otros beneficios, como los frutos del algarrobo y sus derivados para el consumo humano y para el suplemento natural del ganado. El reconocimiento de la tenencia comunitaria de la tierra no puede quedar como un hecho anecdótico o simplemente como una forma de reivindicar socialmente al grupo indígena; debería involucrar las diversas formas de entender e intervenir el territorio que aporten a la cultura de la población local.

La consideración de las transformaciones del territorio en el tiempo y su abordaje desde diferentes escalas es de gran importancia, ya que brinda el marco para comprender las prácticas que actualmente despliegan los pobladores y en consecuencia poder diagnosticar la realidad del sector desde diversas miradas y establecer medidas o políticas que sean coherentes con las características del medio natural y cultural. En este sentido y en alusión a diversos textos que señalan a los pobladores como causantes de la desertificación, en el abordaje de las transformaciones se da cuenta de que los procesos de desertificación en el noreste de Mendoza comenzaron con el aprovechamiento masivo del bosque nativo y la apropiación de agua superficial para la construcción del oasis de forma sostenida desde principios del siglo XX. Desde la consideración de una escala mayor y en sintonía con las transformaciones territoriales se comprende que la configuración habitacional dispersa resulta una respuesta adecuada a las características naturales del territorio, pero que para alcanzar la sustentabilidad indefectiblemente debería haber algún reglamento o acuerdo entre pobladores o la presencia activa del Estado en la regulación del acceso a los bienes naturales disponibles.

Además, queda de manifiesto que al interior del caso de estudio se hace referencia a la sustentabilidad fuerte, ya que el sistema socioeconómico es dependiente del ecosistema natural en una articulación permanente. Reconocer esta situación facilitaría la formulación de políticas y ac- 
ciones por parte del Estado que tiendan a poner en valor la naturaleza siempre en relación con las particularidades de la población local, su capacitación y participación, vinculando conocimientos técnicos y saberes tradicionales.

\section{Agradecimientos}

Este trabajo fue elaborado a partir de las investigaciones realizadas en el marco de la tesis doctoral del autor financiada por el Consejo Nacional de Investigaciones Científicas y Técnicas (CONICET), Argentina.

\section{Referencias}

Abraham, Elena y Prieto, Rosario (1981). "Enfoque diacrónico de los cambios ecológicos y de las adaptaciones humanas en el noreste árido mendocino". Cuadernos del CEIFAR, 8, Mendoza, pp. 109-139.

Abraham, Elena y Prieto, Rosario (1999). "Guanacache, la travesía de los profundos cambios". En Rene Gotthelf (dir.), Guanacache: Fidel Roig Matons, pintor del desierto, Mendoza, EDIUNC, pp. 107-125.

Abraham, Elena y Salomón, Mario (2006). "Indicadores y puntos de referencia de la desertificación utilizados en Argentina por diversos usuarios". En Elena Abraham y Gertjan Beekman (eds.), Indicadores de la Desertificación para América del Sur, Mendoza, IICA BID, pp. 113- 140.

Abraham, Elena y Salomón, Mario (2014). "El desierto como oportunidad: desertificación versus desarrollo sustentable". Ciencia e investigación, 64 (1), Buenos Aires, pp. 59-65.

Abraham, Elena; Matallo, Eitor, y de Lima, José (2013). "Ciencia y desertificación en América Latina”. Zonas Áridas, 15 (2), Perú, pp. 349-360.

Acevedo-Osorio, Álvaro; Angarita Leiton, Arlex; León Durán, Mónica, y Franco Quiroga, Karen (2017). "Sustentabilidad y variabilidad climática: Acciones agroecológicas participativas de adaptación y resiliencia socioecológica en la región alto-andina colombiana". Luna Azul, 44, Colombia, pp. 06-26.

Alfaro, Wilfredo (2005). "Conceptos básicos para el análisis social, económico, ambiental e institucional de la desertificación”. En César Morales y Soledad Parada (eds.), Pobreza, desertificación y degradación de los recursos naturales, Santiago de Chile, CEPAL, pp. 59-88.

Balvanera, Patricia; Astier, Marta; Gurri, Francisco, y Zermeño-Hernández, Isela (2017). "Resiliencia, vulnerabilidad y sustentabilidad de sistemas socioecológicos en México". Revista mexicana de biodiversidad, 88, México, D.F, pp. 141-149.

Benedetti, Alejandro (2011). “Territorio: concepto integrador de la geografía contemporánea”. En Patricia Souto (coord.), Territorio, lugar, paisaje. Prácticas y conceptos básicos en geografía. Buenos Aires: Facultad de Filosofía y Letras, UBA, pp. 11-82. 


\section{Ambiente}

Bifani, Paolo (1999). Medio ambiente y desarrollo sostenible. Madrid: IEPALA, 593 pp.

Chiavazza, Horacio (2008). "Cambios ambientales y sistemas de asentamiento en el árido normendocino. Arqueología en los paleocauces del Río Mendoza” (Tesis doctoral). Buenos Aires: Universidad Nacional de La Plata.

Cony, Mariano (1995). "Reforestación racional de zonas áridas y semiáridas con árboles de múltiples propósitos". Interciencia, 20 (5), Caracas, Asociación Interciencia, pp. 249-253.

Correa Moreno, Diana (2015). "Indicadores de riesgo de desertificación en zonas del Valle de Cauca (Colombia)" (Tesis doctoral). Colombia: Universidad Nacional de Colombia, Palmira (Valle).

D’Amico, Paula; Moreno, Silvia; Pessolano, Daniela, y Accorinti, Carla (2013) "Territorio y reproducción social: herramientas conceptuales para repensar el desierto de Lavalle (Argentina)". Ambiente y Desarrollo, 17 (33), Colombia, pp. 57-70.

Daly, Herman (2007). Ecological economics and sustainable development, selected essays of Hernan Daly. Inglaterra: MPG Books Ltd, 280 pp.

Dimuro Peter, Glenda (2009). "Los ecosistemas como laboratorios. La búsqueda de modos de vivir para una operatividad de la sustentabilidad" (Tesis de master). España: Universidad de Sevilla.

Ejarque, Mercedes (2009). "Hacia un estado de la cuestión sobre las representaciones sociales sobre la desertificación”. En FSOC- UBA. $5^{\circ}$ Jornadas de Jóvenes Investigadores del Instituto de Investigaciones Gino Germani, Buenos Aires, Argentina.

Erazo, Manuela y Garay Flühmann, Rosa (2011). “Tierras secas e identidad. Una aproximación cultural a las prácticas de subsistencia de las comunidades campesinas del semiárido. Provincia de Elqui, Chile". Norte Grande, 50, Santiago de Chile, pp. 45-61.

Espejo, Cayetano (1996). "Sistema de explotación ganadera: notas en torno a su concepto". Lurralde, 9, España, Instituto Geográfico Vasco, pp. 89-104.

Esteves, Matias (2014). "Territorio y Arquitectura: la vivienda vernácula del noreste de Mendoza (Argentina)". Zonas Áridas, 15 (2), Perú, pp. 244-259.

Esteves, Matias (2016). "La interacción vivienda vernácula rural-territorio en tierras secas no irrigadas. Estudio de caso: Lagunas del Rosario, Mendoza, Argentina. (Tesis doctoral). Argentina: Universidad Nacional de San Juan.

FAO/LADA (2011). Evaluación de la Desertificación en Argentina. Resultados del Proyecto LADA/ FAO. Buenos Aires: Editorial FAO/SAyDS, 471 pp.

Galafassi, Guido (2004). Naturaleza, sociedad y alienación. Montevideo: Nordan-Comunidad, 166 pp. Galván-Miyoshi, Yankuic; Masera, Omar, y López-Ridaura, Santiago (2008). "Las evaluaciones de sustentabilidad". En Marta Astier, Omar Masera y Yankuic Galván Miyoshi (coords.). Evaluación de sustentabilidad. Un enfoque dinámico y multidimensional. Valencia: SEAE, pp. 41-58. 
Garay Romero, Ingrid y Cabrero Diéguez, Valentín (2013). "El aspecto social de la desertificación: análisis de la percepción de la administración pública en Centro América". Gestión y Ambiente, 16 (2), Colombia, pp. 37-48.

Granados-Sánchez, Diódoro; Hernández-García, Miguel; Vázquez-Alarcón, Antonio, y Ruiz-Puga, Pablo (2013). "Los procesos de desertificación y las regiones áridas". Chapingo, 19 (1), México, pp. $45-66$.

Grosso Cepparo, Virginia (2013). "Vivir sin agua. Estrategias frente a la escasez en las tierras secas no irrigadas de Lavalle, Mendoza. Entramados y perspectivas, 3 (3), Buenos Aires, pp. 13-37.

Grosso Cepparo, Virginia (2017). "Agua y tierras secas. Lecturas críticas sobre la escasez hídrica en el departamento de Lavalle (Mendoza, Argentina)". Estudios socioterritoriales, 22, Tandil, pp. 27-45.

Gudiño, Elina, (2017). Ordenar el territorio: Un desafío para Mendoza. Mendoza, Argentina: EDIUNC, $470 \mathrm{pp}$.

Guevara, Juan Carlos; Estévez, Oscar, y Torres, Eduardo (1995). "Receptividad de las pasturas naturales de la llanura de Mendoza”. Multiequina, 4, Mendoza, pp. 29-35.

Haesbaert, Rogerio (2006). Territorios Alternativos. San Pablo: Contexto, 173 pp.

Jiménez Herrero, Luis (2002). "La sostenibilidad como proceso de equilibrio dinámico y adaptación al cambio". ICE, 800, España, pp. 65-84.

Katzer, Leticia (2010). "Tierras indígenas, demarcaciones territoriales y gubernamentalización. El caso Huarpe, provincia de Mendoza". Recuperado de http://www.scielo.org.ar/scielo.php?script=sci_arttext\&pid=S1851-16942010000100006\&lng=es\&nrm=iso (Última consulta: 05 de junio de 2018).

Katzer, Leticia; Giménez Zumbo, Pablo; Chiavazza, Horacio; Miranda Gassull, Virginia, y Vélez, Silvina (2017). "Bio-historia del nomadismo y de la producción territorial en el NE de Mendoza: Lectura interdisciplinar desde la ecología, la arqueología y la etnografía”. Relaciones de la sociedad argentina de antropología, 42 (2), Buenos Aires, pp. 345-367.

Leff, Enrique (2007). "La complejidad ambiental". Recuperado de http://journals.openedition.org/ polis/4605 (Última consulta: 07 de junio de 2018).

López, Milagros (2002). "Consolidación y perspectivas de la actividad ganadera en las áreas de montaña de Galicia: La importancia de la explotación extensiva de bovino de carne y ovino". XI Coloquio de geografía rural: los espacios rurales entre el hoy y el mañana, Santander, España.

Maestre Gil, Fernando (2011). "Avances recientes y retos en el estudio y evaluación de la desertificación: de la teoría a la práctica”. Cuides, 7, España, pp. 37-60.

Martínez Alier, Joan (2006). "Los conflictos ecológicos distributivos y los indicadores de sustentabilidad”. Recuperado de http://www.redalyc.org/articulo.oa?id=30551307> (Última consulta: 11 de junio de 2018). 
Martínez, Luis Manuel (2003). "Ganadería extensiva y manejo sustentable de sistemas naturales y agrosilvopastoriles en Manantlán, Jalisco". De vinculación y ciencia, 12, Guadalajara, pp. 14-26.

Masa, Isidro (1990). Toponimia, tradiciones y leyendas mendocinas. Buenos Aires: Fundación Banco de Boston, 315 pp.

Ministerio del Interior, Obras Públicas y Vivienda (MIOPyV) (2014). "Directrices para el Ordenamiento Territorial de las áreas rurales de Mendoza". Mendoza, Argentina, 650 pp.

Miranda Gassull, Virginia y Gómez, Heliana (2016). "Territorio dominado vs territorio apropiado. El caso del norte de la provincia de Mendoza". revIISE, 8 (8), San Juan, pp. 75-88.

Morales, César (2005). "Pobreza, desertificación y degradación de tierras". En César Morales y Soledad Parada (eds.). Pobreza, desertificación y degradación de los recursos naturales. Santiago de Chile: CEPAL, pp. 25-57.

Morales, César (2012). Los costos de la inacción ante la desertificación y degradación de las tierras en escenarios alternativos de cambio climático. Santiago de Chile: ONU/CEPAL, 94 pp.

Naredo, José (2001). "Economía y sostenibilidad. La economía ecológica en perspectiva". Recuperado de http://www.redalyc.org/articulo.oa?id=30500213 (Última consulta 10 de octubre de 2016).

Oliveira, Vladia; Semedo, José, y Oliveira, Huáscar (2014). "Análisis comparativo de los indicadores geofísicos de desertificación de la isla de Santiago-Cabo Verde (África) y en la región de los Inhamuns (Ceará-Brasil). Zonas Áridas, 15 (2), Perú, pp. 226-243.

Organización de las Naciones Unidas (ONU) (1994). "Convención de las Naciones Unidas de lucha contra la desertificación”. París, Francia, 66 pp.

Osorio, Isabel y Oyarce, Nicole (2010) "Análisis socioeconómico de la desertificación en la Región de Coquimbo". Revista interamericana de ambiente y turismo, 6 (1), Talca, pp. 45-52.

Pastor, Gabriela (2005). "Patrimonio, vivienda y agua en el paisaje del noreste mendocino". En Alicia Fernández Cirelli y Elena Abraham (eds.), El agua en Iberoamérica. Uso y gestión del agua en tierras secas. Mendoza: CYTED XII, pp. 79-92.

Pietragalla, Vanina y Corso, Laura (1994). "Prácticas de manejo sustentable de tierras secas: Su importancia para el desarrollo". En Andrés Ravelo, Ana Planchuelo, Elena Abraham y Stella Navone (eds.), Evaluación de la desertificación en Argentina: Resultados. Buenos Aires: FAO/ SAyDS, pp. 337-431.

Prieto, Rosario (1998). "Formación y consolidación de una sociedad en un área marginal del Reino de Chile: La provincia de Cuyo en el Siglo XVII". Anales de Arqueología y Etnología 52-53. Mendoza, Facultad de Filosofía y Letras-UNCuyo, 336 pp.

Raffestin, Claude (2011). Por una geografía del poder. Traducción y notas Yanga Villagómez Velázquez. México: El Colegio de Michoacán, 190 pp. 
Richard Jorba, Rodolfo (2000). "Modelos vitivinícolas en Mendoza (Argentina). Desarrollo y transformaciones en un periodo secular, 1870-2000”. Historia Económica \& Historia de Empresas, 3 (1), Brasil, pp. 111-148.

Roig, Fidel; Roig, Arturo; Roig, Mario; Roig, Virgilio, y Roig, Enrique (1999). Guanacache. Fidel Roig Matóns, pintor del desierto. Mendoza: EDIUNC, 137 pp.

Rosales, Diego (1877). Historia General del Reyno de Chile, Flandes indiano". Valparaíso: Imprenta del Mercurio, $688 \mathrm{pp}$.

Rubio, Cecilia (2015). "Desertificación en el área no irrigada del departamento de Lavalle" (Tesis doctoral). Argentina: Universidad Nacional de Tucumán.

Salas Zapata, Walter; Ríos Osorio, Leonardo, y Álvarez del Castillo, Javier (2012). "Marco conceptual para entender la sustentabilidad de los sistemas socioeconómicos". Ecología austral, 22, Córdoba, pp. 74-79.

Sánchez, Eugeni (1991). Espacio, economía y sociedad. España: Siglo XXI, 229 pp.

Taylor, Steven y Bogdan, Robert (2008). "La entrevista en profundidad". Métodos cuantitativos aplicados 2, Antología. Recuperado de http://www.ceppia.com.co/Herramientas/Herramientas/Meto-cuantitaivos.pdf\#page=192 (Última consulta: 13/05/2017).

Torres, Laura (2008). "Nueva ruralidad en territorios periféricos: los productores caprinos del noreste de Mendoza (Argentina)". Universitas Humanistica, (66), Colombia, Pontificia Universidad Javeriana, pp. 199-218.

Torres, Laura; Abraham, Elena.; Montaña, Elma, y Torres, Eduardo (2005). "Las dimensiones socioeconómicas de la desertificación: avances en la utilización de indicadores. Un ejercicio en el caso de Mendoza, Argentina". En César Morales y Soledad Parada (eds.), Pobreza, desertificación y degradación de los recursos naturales. Santiago de Chile: CEPAL, pp. 215-234.

Torres, Laura; Abraham, Elena; Torres, Eduardo, y Montaña, Elma (2003). "Acceso a los recursos y distribución de la población en tierras secas de Argentina: el caso de Mendoza. Aportes hacia la equidad territorial". Recuperado de http://www.ub.edu/geocrit/sn/sn-148.htm (Última consulta 25 de febrero de 2018).

Troyo Diéguez, Enrique; Mercado Mancera, Gustavo; Cruz Falcón, Arturo; Nieto Garibay, Alejandra; Valdez Cepeda, Ricardo; García Hernández, José, y Murillo Amador, Bernardo (2014). "Análisis de la sequía y desertificación mediante índices de aridez y estimación de la brecha hídrica en Baja California Sur, noroeste de México". Investigaciones Geográficas, 85, México D.F, pp. 66-81.

Editora asociada: Consuelo Lorenzo Monterrubio Recibido: 9 marzo 2018 Aceptado: 26 junio 2018 